\title{
Straddles on ternary semigroups
}

\author{
Phoschanun Ratanaburee ${ }^{\mathrm{a}}$, Thananya Kaewnoi ${ }^{\mathrm{a}}$, Ronnason Chinram ${ }^{\mathrm{a}, \mathrm{b}, *}$ \\ ${ }^{a}$ Department of Mathematics and Statistics, Faculty of Science, Prince of Songkla University, Hat Yai, Songkhla 90110, Thailand. \\ ${ }^{b}$ Centre of Excellence in Mathematics, CHE, Si Ayuthaya Road, Bangkok 10400, Thailand.
}

\begin{abstract}
A ternary semigroup is a nonempty set with a ternary operation satisfy the associative law. In this paper, we define straddles on ternary semigroups and investigate some properties of straddles of ternary semigroups.
\end{abstract}

Keywords: Straddles, ternary semigroups, commutative elements, homomorphisms.

2010 MSC: 20N10, 20M10.

(C)2019 All rights reserved.

\section{Introduction}

A ternary semigroup is a nonempty set equipped with an associative ternary operation. All semigroups are considered to be ternary semigroups. The existence of ternary operations originated from the study of a ternary analogue of Abelian group in 1932 by Lehmer [8]. Then the notion of ternary semigroups came from the problem of Banach who created an example of ternary semigroups which is not reducible to a semigroup. In addition, he conjectured that every ternary semigroup may be extended to reducible to a semigroup (cf. [9]). Los [9] exposed Banach's problem and showed that the operation in the ternary semigroup is an extension of the binary operation satisfying associative law on some nonempty set. Then many properties of ternary semigroups have been extensively studied by many authors. Sioson [14] introduced the ideal of ternary semigroups. Moreover, he defined regular ternary semigroups. Afterwards, the regularity of ternary semigroups was determined in [13] and [1]. Meanwhile, Iampan [7] conceptualized some properties of ideal extensions in ternary semigroups. Dixit and Dewan [5] studied the properties of quasi-ideals and bi-ideals in ternary semigroups. They also proved that every quasi-ideal is a bi-ideal; however, the converse is not true. From 2009-2011, fuzzy, rough, and rough fuzzy ideals and bi-ideals in ternary semigroups were studied in [10] and [12], respectively. After that, Choosuwan and Chinram [4] characterized maximal and minimal quasi-ideals of ternary semigroups. Later, Thongkam and Changphas [16] introduced the concept of two-sided bases of a ternary semigroup, and constructed

\footnotetext{
*Corresponding author

Email address: ronnason.c@psu.ac.th (Ronnason Chinram)

doi: $10.22436 /$ jmcs.019.04.04
}

Received: 2019-03-19 Revised: 2019-05-30 Accepted: 2019-06-15 
the ternary semigroups containing two-sided bases. Bindu et al. [3] introduced the notion of right T-system transitive, T-homomorphism, and semispace in ternary semigroups and characterized its properties. Prime fuzzy bi-ideals in ternary semigroups were studied in [11]. Ansari and Yaqoob [2] discussed the concept of T-rough ternary subsemigroups, T-rough ideals, T-rough bi-ideals, and T-rough interior ideals in ternary semigroups. Interval valued intuitionistic $(\bar{s}, \bar{t})$-fuzzy ideals of ternary semigroups were studied in [17] and interval valued fuzzy ideal extensions of ternary semigroups were studied in [15]. The research on ternary semigroups in many aspects has been creative and interesting. It is significant to note that analogues of the results of ternary semigroups can be obtained from semigroup theory.

Straddles on semigroups were first established by Franklin and Lindsay [6] in 1961. They gave some properties of straddles on semigroups. Furthermore, they examined the nonempty set of straddles on semigroups which is an abelian group. Inspired by the work of these authors, we will reintroduce the straddles on ternary semigroups and investigate some of its properties. The remainder of this paper is organized as follows: in Section 2, we introduce the definition of ternary semigroups and illustrate several examples of ternary semigroups; the properties of straddles on ternary semigroups are provided in Section 3; and a theorem specifying the relationship between homomorphism and straddles on ternary semigroups is suggested in Section 4.

\section{Ternary semigroups}

A ternary semigroup is a nonempty set $T$ together with a ternary operation $(a, b, c) \mapsto[a b c]$ satisfying the associative law

$$
[[a b c] u v]=[a[b c u] v]=[a b[c u v]], \quad \forall a, b, c, u, v \in T .
$$

The following examples show that a ternary semigroup does not necessarily reduce an ordinary semigroup.

Example 2.1 (Banach's Example). Let $T=\{-i, 0, i\}$ be a ternary semigroup under multiplication over complex number. We have that $\mathrm{T}$ is not a binary semigroup under multiplication over complex number.

Example 2.2. Let $\mathbb{Z}^{-}$be a ternary semigroup under multiplication over integer number. We have that $\mathbb{Z}^{-}$ is not a binary semigroup under multiplication over integer number.

An element $e \in T$ is said to be a selfpotent element, if [eee] $=e$. An element $e \in T$ is said to be a unital element of $\mathrm{T}$ if $[\mathrm{eex}]=[\mathrm{xee}]=[\mathrm{exe}]=\mathrm{x}$ for all $\mathrm{x} \in \mathrm{T}$. Then every unital element of $\mathrm{T}$ is a selfpotent element.

Example 2.3. Consider a ternary semigroup $T=\{-i, i\}$ under multiplication over complex number. We have that $\mathrm{T}$ has no selfpotent element and unital element.

Example 2.4. Consider a ternary semigroup $\mathbb{Z}^{-}$under multiplication over integer number. We have that -1 is both a selfpotent element and a unital element.

Example 2.5. Consider a ternary semigroup $T=\{-i, i, 1,-1\}$ under multiplication over complex number. We have that 1 and -1 are both selfpotent elements and unital elements.

Example 2.6. Consider a ternary semigroup $T=\mathbb{Z}_{6}$ under multiplication over integer modulo 6 . We have that $[\overline{2} \overline{2} \overline{2}]=\overline{8}=\overline{2}$ and $[\overline{2} \overline{2} \overline{1}]=\overline{4} \neq \overline{1}$. This implies that $\overline{2}$ is a selfpotent element but not a unital element.

\section{Straddles on ternary semigroups}

Throughout this section, let $\mathrm{T}$ be a ternary semigroup.

Definition 3.1. Let $a, b \in T$. The ordered pair $(a, b)$ is a straddle on $T$ if $[a x b]=x$ for all $x \in T$. 
Example 3.2. Let $e$ be a unital element of $T$. Then $(e, e)$ is a straddle on $T$ which is called a trivial straddle.

Example 3.3. Consider a ternary semigroup $T=\{-i, 0, i\}$ under multiplication over complex number. Note that this ternary semigroup has no unital elements. We have $(i,-i)$ and $(-i, i)$ are nontrivial straddles on T.

Theorem 3.4. If $(\mathrm{a}, \mathrm{b})$ is a straddle on $\mathrm{T}$, then $\mathrm{x}=[\mathrm{a} \times \mathrm{b}]=[\mathrm{abx}]=[\mathrm{xab}]$, for all $\mathrm{x} \in \mathrm{T}$.

Proof. If $(a, b)$ is a straddle on $T$, then $a=[a a b]$ and $b=[a b b]$. Therefore, for any element $x$ in $T$,

$$
x=[a x b]=[[a a b] x b]=[a[a b x] b]=[a b x],
$$

and

$$
x=[a x b]=[a x[a b b]]=[a[x a b] b]=[x a b]
$$

Theorem 3.5. If $(\mathrm{a}, \mathrm{b})$ is a straddle on $\mathrm{T}$, then $(\mathrm{b}, \mathrm{a})$ is a straddle on $\mathrm{T}$.

Proof. Let $x \in \mathrm{T}$. Then $[\mathrm{b} x \mathrm{a}]=[\mathrm{a}[\mathrm{b} \times \mathrm{a}] \mathrm{b}]=[\mathrm{ab}[\mathrm{xab}]]=[\mathrm{abx}]=\mathrm{x}$.

An element $a$ in $T$ is called a commutative element of $T$ if $[a x y]=[x y a]$ for all $x, y \in T$.

Theorem 3.6. If $(\mathrm{a}, \mathrm{b})$ is a straddle on $\mathrm{T}$, then $\mathrm{a}$ and $\mathrm{b}$ are commutative elements of $\mathrm{T}$.

Proof. Assume that $(a, b)$ is a straddle on $T$ and let $x, y \in T$. By Theorem 3.4 and Theorem 3.5, we have

$$
[a x y]=[b[a x y] a]=[[b a x] y a]=[x y a] .
$$

Similarly, $[\mathrm{bxy}]=[\mathrm{xy} b]$.

Let $T$ be a ternary semigroup with unique unital element $e$ and $a \in T$. An element $a^{\prime}$ in $S$ is called a left-inverse of $a$ if $\left[a^{\prime} a e\right]=\left[a^{\prime} e a\right]=\left[e a^{\prime} a\right]=e$.

Theorem 3.7. Let $\mathrm{T}$ be a ternary semigroup with unique unital element e. If $\mathrm{a}$ is a commutative element with left-inverse $\mathrm{a}^{\prime}$, then $\left(\mathrm{a}, \mathrm{a}^{\prime}\right)$ is a straddle on $\mathrm{T}$.

Proof. Let $x \in \mathrm{T}$. Then $\left[\mathrm{axa}^{\prime}\right]=\left[x \mathrm{a}^{\prime} \mathrm{a}\right]=\left[\left[x \mathrm{a}^{\prime} \mathrm{a}\right] e e\right]=\left[x\left[\mathrm{a}^{\prime} \mathrm{ae}\right] e\right]=[x e e]=x$. Hence, $\left(\mathrm{a}, \mathrm{a}^{\prime}\right)$ is a straddle on $\mathrm{T}$.

Theorem 3.8. Let $\mathrm{T}$ be a ternary semigroup with unique unital element e. T has a nontrivial straddle if and only if $\mathrm{T}$ has a commutative element a with left-inverse $\mathrm{a}^{\prime}$ other than the unital element.

Proof. Let $\left(a, a^{\prime}\right)$ be a nontrivial straddle on T. By Theorem 3.5, $\left(a^{\prime}, a\right)$ is a straddle on T. By Theorem 3.4, $\left[a^{\prime} a e\right]=\left[a^{\prime} e a\right]=\left[e a^{\prime} a\right]=e$. Thus $a^{\prime}$ is a left-inverse of $a$. By Theorem 3.6, $a$ is a commutative element of T. Conversely, by Theorem 3.7, $\left(a, a^{\prime}\right)$ is a nontrivial straddle.

An element $a^{\prime}$ in $S$ is called a right-inverse of $a$ if $\left[\mathrm{aa}^{\prime} e\right]=\left[\mathrm{aea}^{\prime}\right]=\left[e a a^{\prime}\right]=e$.

Theorem 3.9. Let $\mathrm{T}$ be a ternary semigroup with unique unital e. If $\mathrm{a}$ is a commutative element of $\mathrm{T}$ with right-inverse $\mathrm{a}^{\prime}$, then $\left(\mathrm{a}, \mathrm{a}^{\prime}\right)$ is a straddle on $\mathrm{T}$.

Proof. Let $x \in T$. Then $\left[a^{\prime} x a\right]=\left[a a^{\prime} x\right]=\left[e e\left[a^{\prime} x\right]\right]=\left[e\left[e a a^{\prime}\right] x\right]=[e e x]=x$. Hence, $\left(a^{\prime}, a\right)$ is a straddle on T. By Theorem 3.5, $\left(a, a^{\prime}\right)$ is also a straddle on T.

Theorem 3.10. Let $\mathrm{T}$ be a ternary semigroup with unique unital element e. T has a nontrivial straddle if and only if T has a commutative element a with right-inverse $\mathrm{a}^{\prime}$ other than the unital element.

Proof. This proof is similar to the proof of Theorem 3.8. 
Theorem 3.11. If $\left(\mathrm{a}_{1}, \mathrm{~b}_{1}\right),\left(\mathrm{a}_{2}, \mathrm{~b}_{2}\right)$ and $\left(\mathrm{a}_{3}, \mathrm{~b}_{3}\right)$ are straddles on $\mathrm{T}$, then $\left(\left[\mathrm{a}_{1} \mathrm{a}_{2} \mathrm{a}_{3}\right],\left[\mathrm{b}_{1} \mathrm{~b}_{2} \mathrm{~b}_{3}\right]\right)$ is a straddle on $\mathrm{T}$.

Proof. Assume that $\left(a_{1}, b_{1}\right),\left(a_{2}, b_{2}\right)$ and $\left(a_{3}, b_{3}\right)$ are straddles on $T$. Let $x \in T$. By Theorem 3.4 and Theorem 3.6,

$$
\begin{aligned}
{\left[\left[a_{1} a_{2} a_{3}\right] x\left[b_{1} b_{2} b_{3}\right]\right] } & =\left[\left[a_{1} a_{2} a_{3}\right] x\left[b_{3} b_{1} b_{2}\right]\right] \\
& =\left[a_{1} a_{2}\left[a_{3} \times b_{3}\right] b_{1} b_{2}\right] \\
& =\left[a_{1}\left[a_{2} x b_{1}\right] b_{2}\right] \\
& =\left[a_{1}\left[x b_{1} a_{2}\right] b_{2}\right] \\
& =\left[\left[a_{1} \times b_{1}\right] a_{2} b_{2}\right] \\
& =\left[x a_{2} b_{2}\right] \\
& =x
\end{aligned}
$$

Corollary 3.12. Let $\mathrm{T}$ be a ternary semigroup with a straddle on $\mathrm{T}$ and $\mathrm{S}(\mathrm{T})$ be the set of straddles on $\mathrm{T}$. Define the ternary operation on $\mathrm{S}(\mathrm{T})$ by

$$
\left[\left(a_{1}, b_{1}\right)\left(a_{2}, b_{2}\right)\left(a_{3}, b_{3}\right)\right]=\left(\left[a_{1} a_{2} a_{3}\right],\left[b_{1} b_{2} b_{3}\right]\right)
$$

for all $\left(\mathrm{a}_{1}, \mathrm{~b}_{1}\right),\left(\mathrm{a}_{2}, \mathrm{~b}_{2}\right),\left(\mathrm{a}_{3}, \mathrm{~b}_{3}\right) \in \mathrm{S}(\mathrm{T})$. Then $\mathrm{S}(\mathrm{T})$ is a ternary semigroup.

Example 3.13. Consider a ternary semigroup $T=\{-i, 0, i\}$ under multiplication over complex number. By previous example, $S(T)=\{(i,-i),(-i, i)\}$.

\section{Relationship between homomorphisms and straddles on ternary semigroups}

Let $T_{1}$ and $T_{2}$ be ternary semigroups. A function $\varphi: T_{1} \rightarrow T_{2}$ is called a homomorphism, if

$$
\varphi([x y z])=[\varphi(x) \varphi(y) \varphi(z)]
$$

for all $x, y, z \in \mathrm{T}_{1}$. Let $\mathrm{Hom}_{\mathrm{T}}$ be the set of all homomorphisms on $\mathrm{T}$. Then Hom $\mathrm{T}$ is a semigroup under composition.

Example 4.1. Let $T$ be a ternary semigroup and $a, b \in T$. Define a function $T_{a, b}: T \rightarrow T$ by

$$
\mathrm{T}_{\mathrm{a}, \mathrm{b}}(\mathrm{x})=\mathrm{axb}
$$

for all $x \in T$. If $(a, b)$ is a straddle of $T$, then $T_{a, b}$ is a homomorphism.

Theorem 4.2. Let $\mathrm{T}$ be a ternary semigroup and $\mathrm{a}, \mathrm{b} \in \mathrm{T}$. Then $(\mathrm{a}, \mathrm{b})$ is a straddle of $\mathrm{T}$ if and only if $\mathrm{T}_{\mathrm{a}, \mathrm{b}}=\mathrm{id} \mathrm{d}_{\mathrm{T}}$.

Proof. This follows from Definition 3.1.

Note that for all $a, b, c, d \in T$, we have

$$
\left(\mathrm{T}_{\mathrm{a}, \mathrm{b}} \circ \mathrm{T}_{\mathrm{c}, \mathrm{d}}\right)(\mathrm{x})=\mathrm{T}_{\mathrm{a}, \mathrm{b}}\left(\mathrm{T}_{\mathrm{c}, \mathrm{d}}(\mathrm{x})\right)=\mathrm{T}_{\mathrm{a}, \mathrm{b}}([\mathrm{c} x \mathrm{~d}])=[\mathrm{a}[\mathrm{c} x \mathrm{~d}] \mathrm{b}]
$$

for all $x \in T$. For $a, b, c, d \in T, T_{a, b} \circ T_{c, d}$ is denoted by $T_{a c, d b}$. By Theorem 4.2, if $(a, b)$ and $(c, d)$ are straddles on $T$, then $T_{a c, d b}=i d_{T}$. Let $T^{\prime}$ be a free semigroup on $T$, that is,

$$
\mathrm{T}^{\prime}=\left\{\mathrm{a}_{1} \mathrm{a}_{2} \ldots \mathrm{a}_{\mathrm{n}} \mid \mathrm{n} \in \mathbb{N} \text { and } \mathrm{a}_{\mathrm{i}} \in \mathrm{T}\right\} .
$$

Define a binary operation $*$ on $T^{\prime} \times T^{\prime}$ by $(a, b) *(c, d)=(a c, d b)$ for all $a, b, c, d \in T^{\prime}$. Then $\left(T^{\prime} \times T^{\prime}, *\right)$ is a semigroup and $\bar{T}=\left\{(a, b) \in T^{\prime} \times T^{\prime} \mid T_{a, b}=i d_{T}\right\}$ is a subsemigroup of $T^{\prime} \times T^{\prime}$. 


\section{Acknowledgment}

This paper was supported by Algebra and Applications Research Unit, Faculty of Science, Prince of Songkla University.

\section{References}

[1] M. Y. Abbasi, S. A. Khan, On some generalized ideals in ternary semigroups, Quasigroups Related Systems, 25 (2017), 181-188. 1

[2] M. A. Ansari, N. Yaqoob, T-rough ideals in ternary semigroups, Int. J. Pure Appl. Math., 86 (2013), 411-424. 1

[3] P. Bindu, S. Yella, M. Rao Dasari, T-systems in ternary semigroups, Ital. J. Pure Appl. Math., 34 (2015), 221-224. 1

[4] P. Choosuwan, R. Chinram, A study on quasi-ideals in ternary semigroups, Int. J. Pure Appl. Math., 77 (2012), $639-647$. 1

[5] V. N. Dixit, S. Dewan, A note on quasi and bi-ideals in ternary semigroups, Internat. J. Math. Math. Sci., 18 (1995), 501-508. 1

[6] S. P. Franklin, J. W. Lindsay, Straddles on semigroups, Math. Mag., 34 (1960/61), 269-270. 1

[7] A. Iampan, Some properties of ideal extensions in ternary semigroups, Iran. J. Math. Sci. Inform., 8 (2013), 67-74. 1

[8] D. H. Lehmer, A ternary analogue of abelian groups, Amer. J. Math., 54 (1932), 329-338. 1

[9] J. Loś, On the extending of models (I)*, Fund. Math., 42 (1955), 38-54. 1

[10] P. Petchkhaew, R. Chinram, Fuzzy, rough and rough fuzzy ideals in ternary semigroups, Int. J. Pure Appl. Math., 56 (2009), 21-36. 1

[11] A. M. Rezvi, J. Mehmood, On prime fuzzy bi-ideals in ternary semigroups, Mat. Vesnik, 64 (2012), 79-96. 1

[12] S. Saelee, R. Chinram, A study on rough, fuzzy and rough fuzzy bi-ideals of ternary semigroups, IAENG Int. J. Appl. Math., 41 (2011), 172-176. 1

[13] M. L. Santiago, S. Sri Bala, Ternary semigroups, Semigroup Forum, 81 (2010), 380-388. 1

[14] F. M. Sioson, Ideal theory in ternary semigroups, Math. Japon., 10 (1965), 63-84. 1

[15] S. Suebsung, R. Chinram, Interval valued fuzzy ideal extensions of ternary semigroups, Int. J. Math. Comput. Sci., 13 (2018), 15-27. 1

[16] B. Thongkam, T. Changphas, On two-sided bases of ternary semigroups, Quasigroups Related Systems, 23 (2015), 319-324. 1

[17] N. Yaqoob, M. Khan, M. Akram, A. Khan, Interval valued intuitionistic $(\overline{\mathrm{s}}, \overline{\mathrm{t}})$-fuzzy ideals of ternary semigroups, Indian J. Sci. Technol., 6 (2013), 5418-5428. 1 